\title{
Mobile Computed Tomography in the Trauma/Critical Care Environment: Preliminary Clinical Experience
}

Stuart E. Mirvis, M.D., Kathirkamaganathan Shanmuganathan, M.D., Richard Donohue, R.T.R., William H. White, R.T.R., Steven Fritz, Ph.D., and Robbi Hartsock, R.N.

Department of Radiology, University of Maryland Medical System,

Baltimore, Maryland

This study reviews the initial clinical experience using a portable computed tomographic (CI) scanner in the trauma resuscitation unit (TRU), intensive care units (ICUs), and operating rooms (ORs) of a large trauma center. Data were collected on the first 200 patients scanned within the trauma center (including 92 in the TRU, 92 in the ICUs, and 16 in the ORs) over the first 5 months of operation. Evaluation forms were completed by interpreting radiologists, $C T$ technicians operating the system, and nurses/clinicians involved with patients being scanned. Information sought included subjective image quality, ease of use, software and hardware limitations, accessibility to and monitoring of patients during scanning, mobility, and perceived advantages or limitations compared to

\section{Key Words}

Computed tomography; Cost-effectiveness; Radiation exposure; Trauma; Critical/intensive care

Address correspondence and reprint requests to: Stuart E. Mirvis, M.D., Department of Radiology, University of Maryland Medical System, 22 South Greene Street, Baltimore, MD 21201.

1070-3004/97/212-217/\$3.00 Volume 4, Number 4 American Society of Emergency Radiology (C) 1997 fixed CT. Radiation scatter also was measured to determine the safety of the scanner for portable use.

CT scans of 224 body regions performed in 200 patients included: cranium (163), cervical spine (21), abdomen/pe/vis (14), chest (12), face (8), thoracic/lumbar spine (4), and temporal bone (2). Image quality was judged subjectively as excellent (107), good (99), poor (6), or artifact degraded (12). Patient transfer to the scanner was judged to be easy (63) or fair/difficult (4). Patient accessibility and monitoring during scanning was assessed as easy (61) or fair/difficult (6). Access in the smaller TRU bays was more difficult than in the ICU or OR. Cited advantages over fixed CT included decreased risk and cost of transport, quicker assessment of cranial injury on admission, and more rapid postcraniotomy assessment. Limitations cited were relatively slow scan speed, slightly poorer image quality, and technical limits on scanning multiple body regions. Radiation scatter was $25 \%$ of the ALARA (as Iow as reasonably achievable) target for the scanner operator. Initial clinical experience with mobile $C T$ in the $T R U, I C U$, and $O R$ of a major trauma center shows application for focused admission scanning, assessment of critically ill ICU patients, and immediate postoperative assessment after craniotomy. The system has been incorporated easily into the trauma center and has been accepted enthusiastically. Further technical improvements are anticipated to extend the range of applications in this clinical setting.

A mobile computed tomographic (CT) scanner, Tomoscan-M, was introduced into our trauma center in mid-1996. During the first 5 months of operation, the scanner was used in three locations, including the shock-trauma center trauma resuscitation unit (TRU) (Fig. 1), the 72-bed trauma center intensive care units (ICUs) (Fig. 2), and the five trauma center operating rooms (ORs) (Fig. 3). The purpose of this report is to describe our early experience with mobile $\mathrm{CT}$ in these environments. The report is based on the evaluations of radiologists, CT technologists, nurses, and traumatology physicians. In addition, radiation scatter associated with the mobile CT scanner was assessed by our department chief physicist to assess the safety of the scanner as a mobile imaging device. 


\section{MATERIALS AND METHODS}

\section{CT system parameters}

The Tomoscan-M mobile CT scanner is manufactured by Analogic Corp. (Peabody, MA) and marketed in the United States by Philips Medical Systems (Shelton, CT). There is a current world-wide installed base of greater than 100 units. The scanner has three main components: a gantry on wheels (weight $=1000$ pounds), a CT table on wheels (weight $=$ 300 pounds) that docks to the CT gantry, and an operator's console. A mobile power CT-injector, Envision (Medrad, Inc., Pittsburgh, PA), has been specially designed for transport as a single-wheeled unit for use with the mobile CT system.

\section{Figure 1}

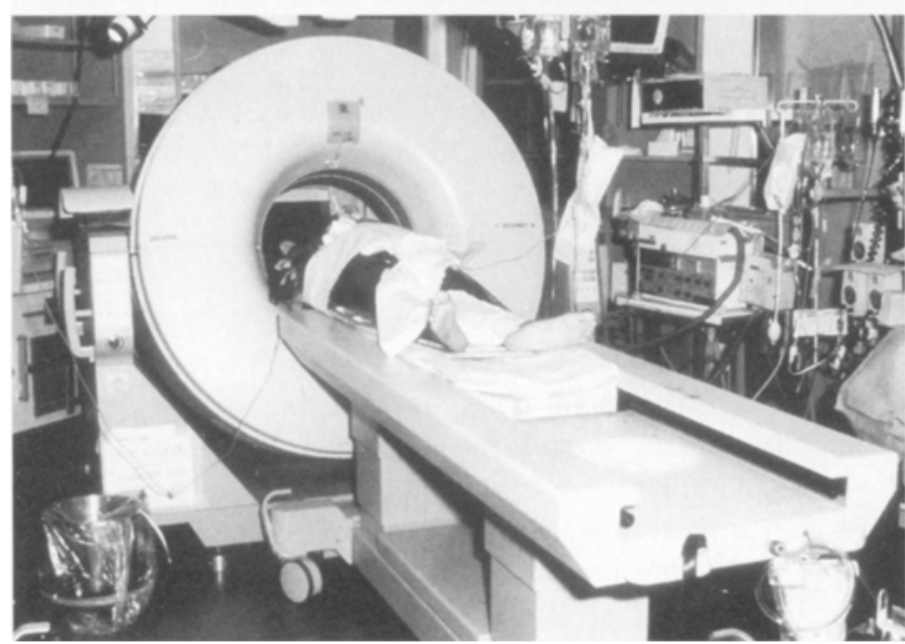

Figure 1. Patient undergoing emergent cranial scanning with mobile CT in the admitting area of the trauma center.

\section{Figure 2}

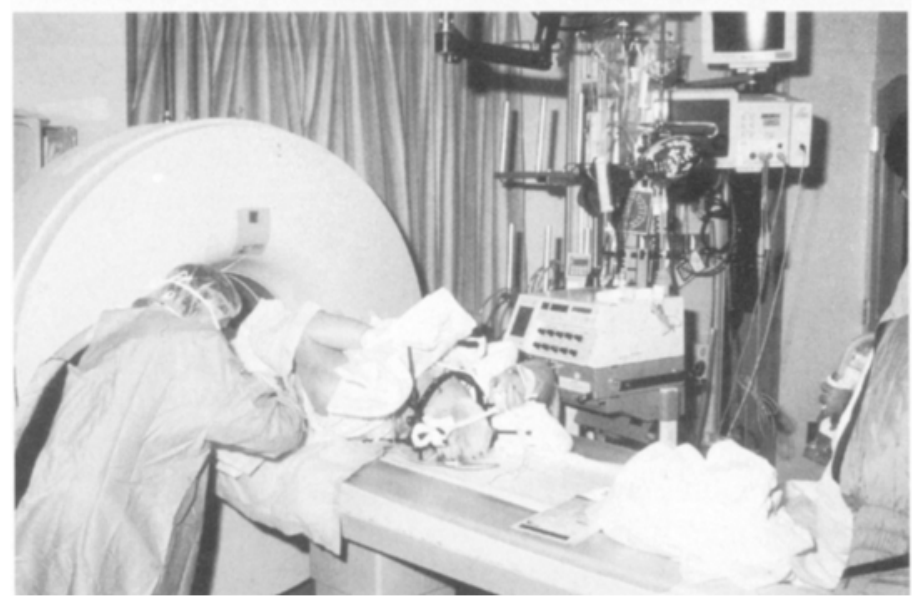

Figure 2. Critical care patient undergoing percutaneous mobile CT-guided drainage of an abdominal abscess in the intensive care unit room without potential interruption of physiologic support or intensive monitoring.
The CT gantry has a $60-\mathrm{cm}$ aperture $(46-\mathrm{cm}$ field-ofview) and uses 2, 4, and $6 \mathrm{sec}$ scan times with $5 \mathrm{sec}$ reconstruction time. Typical scan technique is $50 \mathrm{~mA}$ at 120 $\mathrm{kVp}$ for $2 \sec (100 \mathrm{~mA})$ for the chest, head, and abdomen/pelvis, and $4 \mathrm{sec} / \mathrm{scan}(200 \mathrm{~mA})$ for the spine. The system typically requires about one-third of the milliamperes used for conventional CT owing to a combination of detector-tube distance and high detector efficiency. The gantry tilts $+30^{\circ}$ and $-25^{\circ}$ and has the unique ability to translate in the horizontal axis up to 35 $\mathrm{cm}$, allowing head, neck, and face scanning without patient transfer to the CT bed. The gantry is transportable on elevators and through standard 3-foot doorways and requires two persons for safe navigation.

The CT scanner has a 1300-mm metal-free scanning range, supports bidirectional scanning, and has a weight limit of 350 pounds. The operator's console uses a Windows-based interface on a SPARC 5 computer. Autovoice, autofilming, and erasable optical disk storage for $1300512^{2}$ images is provided. The system disk can store $1200512^{2}$ images. The console interfaces directly over most standard laser cameras for hardcopy generation or over a network using a DICOM standard. Software to support volume scanning is in development.

System power is provided by four 48 -volt batteries that charge from any standard $20 \mathrm{amp} / 115$ volt or 220 volt outlet. At full charge, the system can perform approximately 160 images at $100 \mathrm{~mA}$ before recharging. A full recharge is accomplished in about 60 minutes and in less than 10 minutes after a typical 10 - to 20 -slice scan.

\section{CT system performance evaluation}

CT performance was assessed using questionnaires provided to interpreting radiologists, CT technologists, nurses, and physicians involved in using the mobile CT

\section{Figure 3}

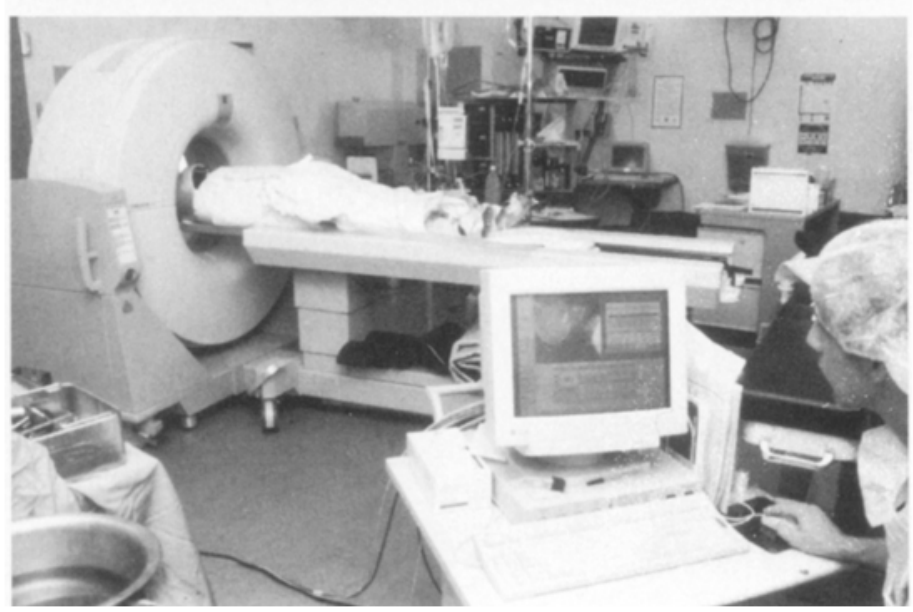

Figure 3. Mobile CT scanner in use in the operating room immediately after evacuation of an epidural hematoma sustained from blunt head trauma. $A$ delayed bleed (Fig. 4) necessitated immediate repeat craniotomy on the opposite side. 

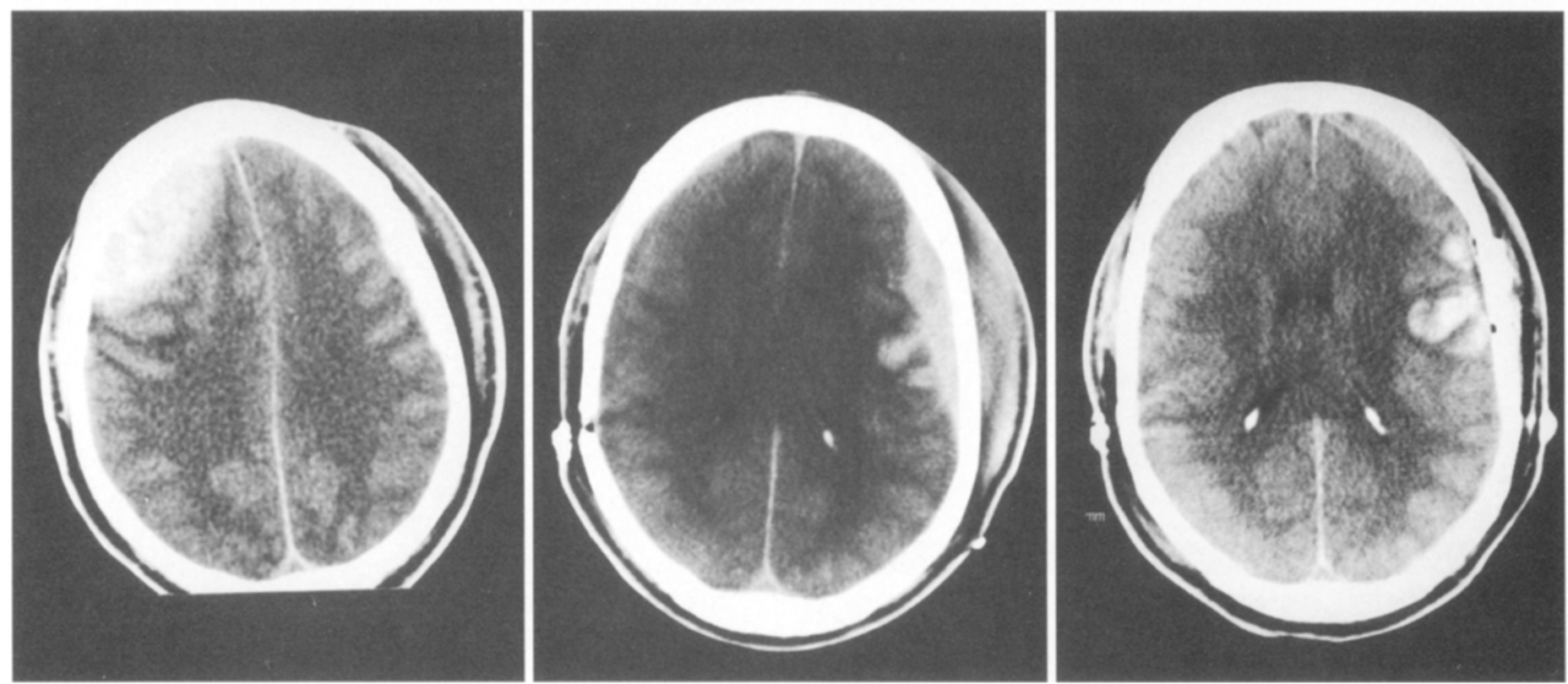

Figure 4. Delayed intracerebral hematoma. Left, initial CT scan performed on fixed CT on the patient depicted in Figure 3 shows a right frontal epidura hematoma, thin left subdural collection, thin parafalx subdural blood, and left frontoparietal scalp contusion at the impact site. Center, mobile CT scan obtained in the OR immediately after evacuation of the epidural collection shows expansion of the left-sided subdural hematoma and underlying cortical contusions. Right, after repeat left-sided craniotomy, the patient had a repeat study on the fixed scanner; it showed successful evacuation of the left subdural collection with mild residual mass effect.

system. Radiologists were asked to judge image quality subjectively as poor, good, or excellent. Poor images were considered unreliable for interpretation; good images were considered diagnostic, but inferior in diagnostic quality to the trauma center's fixed CT system (Somatom Plus 4; Siemens Medical Systems, Iselin, NJ); and excellent scans were considered diagnostically equivalent to fixed CT. Artifacts and scanning technique were recorded for all cases. Whenever possible, direct comparison was made to a CT of the same body region performed within $24-48$ hours on the fixed spiral CT system. CT technologists were asked to record any scanner problems, related to either hardware or software, and ease of system interface learning and operation. Nurses and clinical physicians were asked to assess the system for safety, patient access and monitoring during scanning, ease of patient transport to the CT bed, and advantages and disadvantages of the mobile system compared with fixed CT. All responses were tabulated by two of the authors (S. E. M., $\mathrm{K}$. S.), who also interpreted the scans in most patients.

Radiation scatter was measured at various points around the mobile CT scanner using a Victoreen Panoramic Survey Meter (Victoreen, Inc., Cleveland, $\mathrm{OH}$ ). Scatter was assessed while scanning a $30-\mathrm{cm}^{2}$ plastic phantom using a $100-\mathrm{mA}$ exposure.

\section{RESULTS}

In the first 5 months of operation, the mobile CT scanner performed 224 scans in 200 patients, including the cranium
(163), the cervical spine (21), the abdomen/pelvis (14), the chest (12), the face (8), the thoracic or lumbar spine (4), and the temporal bone (2). Image quality was judged as excellent in 107 studies, good in 99, poor in 6, and severely compromised by artifacts from external objects (intraventricular catheters, intracranial pressure monitors, metal scalp sutures, etc.) in 12 . It was found that cranial scans at 2 $\sec (100 \mathrm{~mA})$ were too noisy and more compromised by artifacts from extracranial monitors. Some small foci of hemorrhage were not consistently seen when compared with contemporaneous scans of the cranium performed on the fixed CT scanner (1.5-sec scan, $120 \mathrm{kVp}, 200 \mathrm{~mA}$ ). After increasing cranial scan technique to $200 \mathrm{~mA}$ (4 sec scan time), there was a significant improvement in image quality, with $34(51 \%)$ of 63 additional head scans being judged excellent and 29 (49\%) judged good, without any poor or uninterpretable studies. No significant increase in motioninduced artifacts was noted using the $4 \mathrm{sec}$ scan time. Spine images were satisfactory at $4 \mathrm{sec}, 50 \mathrm{~mA}$. Image quality was considered diagnostic in all other body regions using $2 \mathrm{sec}$ scan time and $50 \mathrm{~mA}$.

CT technologists using the system found the Windowsbased CT interface to be easy to learn and operate. The CT gantry required two persons to move and guide it safely. The original gantry wheels could become stuck over the short gap entering elevators, and a redesign to larger wheels was suggested and rapidly implemented. A recurring problem with system "time-outs" was encountered and corrected with a system software update. 

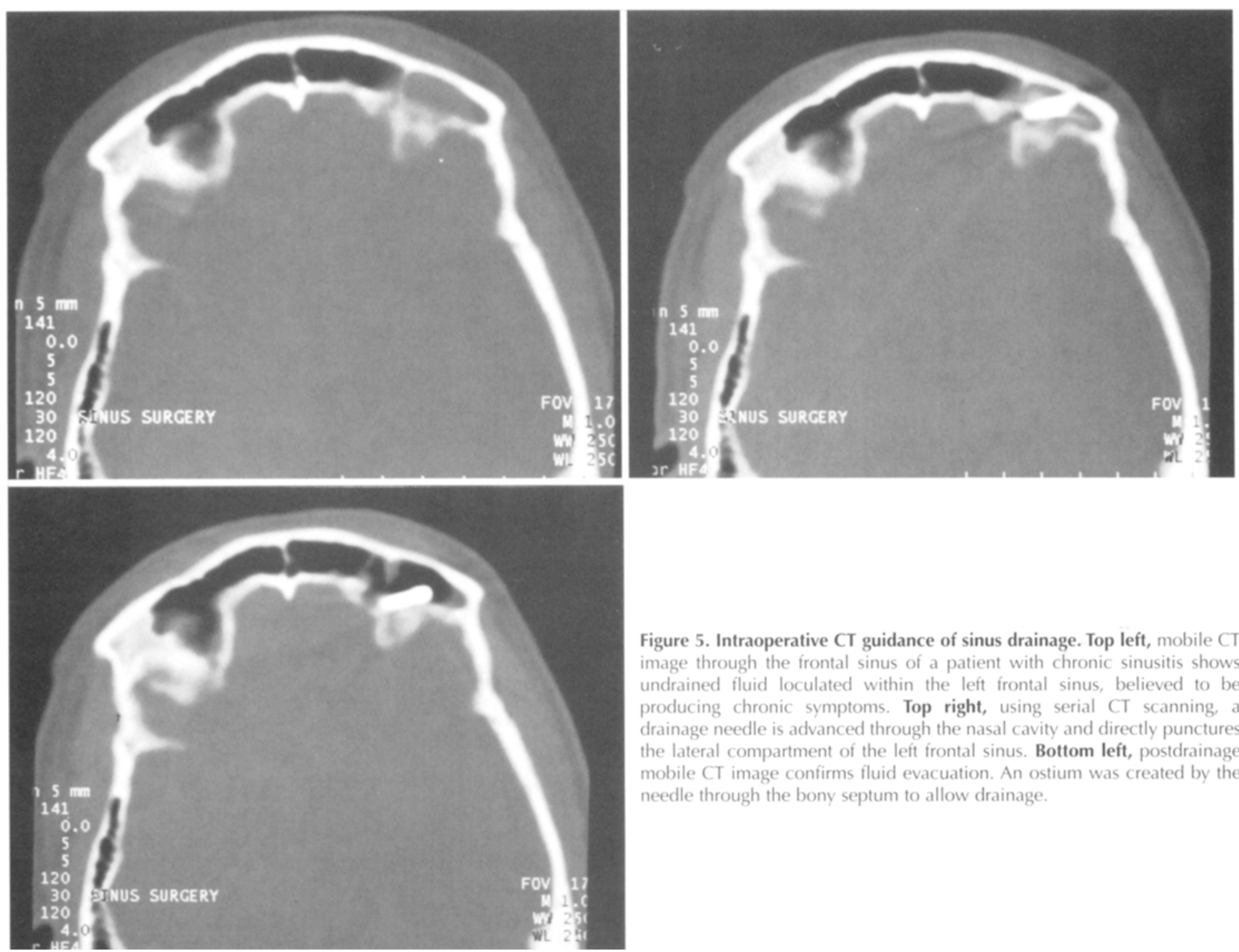

Figure 5. Intraoperative CT guidance of sinus drainage. Top left, mobile CT image through the frontal sinus of a patient with chronic sinusitis shows undrained fluid loculated within the left frontal sinus, believed to be producing chronic symptoms. Top right, using serial CT scanning, a drainage needle is advanced through the nasal cavity and directly punctures the lateral compartment of the left frontal sinus. Bottom left, postdrainage mobile CT image confirms fluid evacuation. An ostium was created by the needle through the bony septum to allow drainage.

Nurses and physicians involved with patients being scanned on the system evaluated 67 patients on the written survey during the assessment period. Movement of patients to the CT bed using traditional transfer methods (backboard, air-cushion device, or plastic slide) was judged easy in 63 patients and fair/difficult in 4 patients. Access to the patient and monitoring of physiologic parameters was assessed as easy in 61 and fair/difficult in 6. The most significant problem encountered was access to the head of the patient by anesthesia personnel with the scanner in the resuscitation unit bay (dimensions $=13 \mathrm{ft} 4$ in width, $11 \mathrm{ft} 2$ in length) (Fig. 1).

The mobile CT scanner was utilized in the operating room for 16 patients after craniotomy for extra-axial hematomas. In three cases, a postoperative new or significantly increased "delayed" intracranial hemorrhage was observed (Fig. 4). All three patients went back to the operating table for repeat craniotomy with evacuation of delayed bleeds. In all cases, the original sterile field and instruments were utilized without a need to use additional OR resources.

Exposure rates from radiation scatter were determined at eight locations around the CT scanner. Using a model of 50 scans/week, 20 slices/scan, and $2 \mathrm{sec}$ scan time at $50 \mathrm{~mA}$, the operator (3-4 meters from gantry) would receive 26.572 $\mathrm{mR} /$ month $[25 \%$ of the ALARA (as low as reasonably achievable) target]. Personnel standing at 1 meter from the foot of the CT gantry for all scans performed during a month would receive $96.625 \mathrm{mR} /$ month, just below the ALARA target. Since the CT scanner moves from site to site each day, no site has more than one or two scans performed per day with the same personnel attending the patient at that site, and all personnel are restricted to a distance from the gantry beyond the CT technologist for patient monitoring, the authors do not believe that radiation monitoring is indicated for personnel other than the CT technologist. 


\section{DISCUSSION}

CT has a major diagnostic role to play in evaluating trauma, emergency room, and critically ill patients. Transport of critically ill patients to the CT scan room, however, may engender difficulties for both the patient and the hospital staff $(1-4)$. Critically ill patients may be only marginally stable physiologically, requiring precise and ongoing monitoring of physiologic parameters, which could be compromised by movement from the critical care environment to the CT scanner (1-5). Some critical care patients receive continuous hemofiltration, extracorporeal lung assist, aortic counterpulsation balloon support, or other intensive interventions that make travel for CT virtually impossible. Movement of patients receiving invasive monitoring and intensive care support, including the respiratory and circulatory systems, requires many specially trained personnel, such as respiratory therapists, nurses, and physicians, as well as considerable time to optimize safe transport for CT scanning when indicated. Requiring these personnel to leave the ICU floor decreases coverage for other ICU patients and is associated with significant cost per patient transport $(5,6)$. In 1988, Indeck et al. (5) reported a hospital cost of $\$ 465$ incurred per patient transport from the ICU in the Maryland Shock-Trauma Center, and in 1992, Hurst et al. (6) reported an average hospital cost of $\$ 452$ for each patient transported from the ICU for diagnostic studies. Costs are related to personnel, disposable equipment used in transport, and the amortized cost of mobile monitoring equipment $(5,6)$. Finally, the time required to transfer and scan critically ill patients may decrease the availability of CT for other patients, including those requiring acute $\mathrm{CT}$ scanning.

The development of delayed intracerebral hemorrhage is a known complication of craniotomy to evaluate extra-axial hematomas $(7,8)$. Delayed hemorrhages are believed to occur as a result of decreased tamponade effects secondary to a sudden decrease in intracranial pressure after surgery. Among the first 16 patients having immediate cranial CT after craniotomy for extra-axial hematoma, three had significant new intracerebral hemorrhages identified (Fig. 4). Typically, in our center, patients may not have postoperative CT for several hours after craniotomy unless significant neurologic deterioration is evident after surgery. In those cases in which a new intracerebral or extra-axial bleed was seen, all three patients were returned to the OR table without the need to prepare the OR a second time, decreasing costs associated with another craniotomy. A prospective study assessing the value of immediate postcraniotomy head CT is now ongoing in our center.

The value of mobile $C T$ in the admitting area of the trauma center was less obvious. Although patients were directly admitted to the CT bed and scanned after protocol radiographs were obtained, we did not appreciate a significant time or cost benefit. In our center, the fixed CT is directly adjacent to the admitting area and has full monitoring and patient support capability. The relatively slow speed of the mobile system compared with that of the fixed spiral CT offset the time difference in initiating the CT scan more quickly on the mobile system. In addition, multiple body regions frequently are requested by the trauma clinical staff for the initial CT evaluation. The relative slowness of the mobile CT scanner and the need for recharge or tube cooling are compounded when multiple body regions are scanned at one time. In the acute setting, the mobile CT scanner is best applied for one- or two-region scanning (for example, when a patient is admitted with known major head or spine injury as the primary insult). The presence of the mobile CT scanner in the trauma admitting area was useful when multiple patients requiring urgent CT scans (typically cranial scans) were admitted in close succession or when the fixed CT system was not operating.

Recently, we have begun to utilize the CT scanner to assist intraoperatively in neurosurgical and ear, nose, and throat cases, as well as in conjunction with cerebral angiography. Use of intraoperative CT in a recent case (Fig. 5) was believed to have saved both on intraoperative time and on the extent of surgery required. Investigation of the intraoperative applications of mobile CT is the subject of a future report.

\section{CONCLUSION}

Mobile CT has proven very useful in the trauma/critical care setting, with very positive acceptance by radiology and clinical care staff. In the ICU setting, the system permits CT studies both in patients who cannot travel and in those who are at significant transport risk owing to physiologic instability. The decreased need for ICU patient transport improves the availability of ICU staff, increasing efficiency and decreasing the cost associated with the transport of ICU patients. Excessively long patient transfer time within the fixed scanning suite is also decreased, improving patient throughput and improving scanner availability for patients needing acute CT. Initial experience suggests that mobile CT, at its current state of technology, serves best as a singleor two-body region scanner in the emergency admitting area but can provide back-up support for a fixed system when several patients need urgent CT. Finally, the ability to scan patients immediately after craniotomy without leaving the OR appears beneficial but requires further detailed study. The potential for mobile CT to guide certain intraoperative procedures or to assess outcomes immediately after other surgical procedures is currently being investigated.

\section{ACKNOWLEDGMENT}

The authors would like to express their appreciation to David Crandall for photographic support.

\section{REFERENCES}

1. Bramen SS, Dunn SM, Amico CA, Millman RP. Complications of intrahospital transport in critically ill patients. Ann Intern Med $1987 ; 107: 469-73$. 
2. Smith I, Fleming S, Cernaianu A. Mishaps during transport from the intensive care unit. Crit Care Med 1990;18:278-81.

3. Andrews PJD, Piper IR, Dearden NM, Miller JD. Secondary insults during intrahospital transport of head-injured patients. Lancet $1990 ; 335 ; 327-30$.

4. Weg JG, Haas CF. Safe intrahospital transport of critically ill ventilator-dependent patients. Chest 1989;96:631-5.

5. Indeck M, Peterson S, Smith J, Brotman S. Risk, cost, and benefit of transporting ICU patients for special studies. J Trauma 1988;28:1020-5.

6. Hurst JM, Davis K Jr, Johnson DJ, Branson RD, Campbell RS, Branson PS. Cost and complications during in-hospital transport of critically-ill patients: a prospective cohort study. J Trauma $1990 ; 33: 582-5$.

7. Alvarez-Sabin J, Turon A, Lozano-Sanchez M, Vasquez J, Codina A. Delayed posttraumatic hemorrhage "Spat-Apoplexie." Stroke $1995 ; 26: 1531-5$.

8. Stein SC, Spettell C, Young G, Ross SE. Delayed and progressive brain injury in closed-head trauma: radiologic demonstration. Neurosurgery 1993;32:25-31.

\section{Reviewer's Commentary}

Having first seen this instrument at the last ASER meeting, I enjoyed reading this article. Mobile CT obviously represents an extension of this critical tool into settings where patients requiring the use of $\mathrm{CT}$ scanning would not otherwise get it until after considerable delay, as well as inconvenience to many members of the health care team.

I fear only one downside here: the abuse of the unit. As CT becomes even easier and more accessible, the demand for its immediate implementation may require one scanner stationed in the emergency department or trauma unit, one or more in the ICUs, and another one dedicated to the OR. Technically, the unit performs in an acceptable manner. Only the cost of the unit and the cost of more or less continuous service seem to be incompletely explored. As indicated in the title, these results are preliminary, and we anxiously await reports of more extensive experience with mobile $\mathrm{CT}$, both from this institution and elsewhere. 